\title{
Functional Network Architecture Predicts Psychologically Mediated Analgesia Related to Treatment in Chronic Knee Pain Patients
}

\author{
Javeria Ali Hashmi, ${ }^{1,2,4}$ Jian Kong, ${ }^{1,2,4}$ Rosa Spaeth, ${ }^{1}$ Sheraz Khan, ${ }^{2,3,4,5}$ Ted J. Kaptchuk, ${ }^{6}$ and Randy L. Gollub ${ }^{1,4}$ \\ ${ }^{1}$ Department of Psychiatry, Massachusetts General Hospital, Charlestown, Massachusetts 02129, ${ }^{2}$ Harvard Medical School, Boston, Massachusetts 02115, \\ ${ }^{3}$ Department of Neurology and ${ }^{4}$ A. A. Martinos Center for Biomedical Imaging, Department of Radiology, Massachusetts General Hospital, Charlestown, \\ Massachusetts 02129, ${ }^{5}$ Massachusetts Institute of Technology, Cambridge, Massachusetts 02139, and ${ }^{6}$ Program in Placebo Studies, Beth Israel Deaconess \\ Medical Center, Harvard Medical School, Boston, Massachusetts 02215
}

Placebo analgesia is an indicator of how efficiently the brain translates psychological signals conveyed by a treatment procedure into pain relief. It has been demonstrated that functional connectivity between distributed brain regions predicts placebo analgesia in chronic back pain patients. Greater network efficiency in baseline brain networks may allow better information transfer and facilitate adaptive physiological responses to psychological aspects of treatment. Here, we theorized that topological network alignments in resting state scans predict psychologically conditioned analgesic responses to acupuncture treatment in chronic knee osteoarthritis pain patients $(n=45)$. Analgesia was induced by building positive expectations toward acupuncture treatment with verbal suggestion and heat pain conditioning on a test site of the arm. This procedure induced significantly more analgesia after sham or real acupuncture on the test site than in a control site. The psychologically conditioned analgesia was invariant to sham versus real treatment. Efficiency of information transfer within local networks calculated with graph-theoretic measures (local efficiency and clustering coefficients) significantly predicted conditioned analgesia. Clustering coefficients in regions associated with memory, motivation, and pain modulation were closely involved in predicting analgesia. Moreover, women showed higher clustering coefficients and marginally greater pain reduction than men. Overall, analgesic response to placebo cues can be predicted from a priori resting state data by observing local network topology. Such low-cost synchronizations may represent preparatory resources that facilitate subsequent performance of brain circuits in responding to adaptive environmental cues. This suggests a potential utility of network measures in predicting placebo response for clinical use.

Key words: brain network; chronic pain; placebo; predictive analysis; resting state; synchronization

\section{Introduction}

Placebo research shows a direct engagement of the brain in rendering psychological cues, such as positive bias toward a treatment, into analgesic response (Benedetti et al., 2005). Understanding how intricately connected brain networks process psychological cues associated with sham or real treatments and translate them into a diminished experience of pain is an important scientific challenge that has implications for new drug discovery and improved pain therapeutics (Enck et al., 2013).

The brain is functionally a set of network systems that interact to execute a particular psychobiological function (Egúiluz et al.,

\footnotetext{
Received July 25, 2013; revised Jan. 17, 2014; accepted Jan. 24, 2014

Author contributions: J.K., T.J.K., and R.L.G. designed research; J.A.H., R.S., J.K., and R.L.G. performed research; J.A.H. and S.K. contributed unpublished reagents/analytic tools; J.A.H. analyzed data; J.A.H. wrote the paper.

Funding and support for this study came from R01AT005280 (R.L.G. and T.J.K.), R01AT006364 (J.K.), and P01AT002048 to Bruce Rosen (Massachusetts General Hospital, Charlestown, MA). S.K. is funded by Nancy Lurie Marks Family Foundation Fellowship. We thank Irving Kirsch and Mark Vangel for their valuable suggestions and comments.

The authors declare no competing financial interests.

Correspondence should be addressed to either Javeria Ali Hashmi or Randy L. Gollub, 120 Second Avenue, Suite 103, Charlestown, MA 02129. E-mail: javeria.hashmi@utoronto.ca or rgollub@partners.org.

DOI:10.1523/JNEUROSCI.3155-13.2014

Copyright $\odot 2014$ the authors $\quad 0270-6474 / 14 / 343924-13 \$ 15.00 / 0$
}

2005; Bullmore et al., 2009; Biswal et al., 2010). New studies have now offered several clues that baseline blood oxygenation leveldependent (BOLD) activity and functional connectivity in discrete brain regions provide a priori predictions of placebo analgesia induced by subsequent treatment (Wager et al., 2011; Hashmi et al., 2012; Kong et al., 2013). For instance, in healthy subjects, placebo task-activated regions were also activated during the baseline period (Wager et al., 2004; Watson et al., 2009). These baseline activations were later shown to predict placebo analgesia (Wager et al., 2011). Task-localized regions have also shown greater baseline connectivity in chronic back pain patients that had participated in a clinical trial (Hashmi et al., 2012). In these patients, synchronization of prefrontal regions with widespread brain regions particularly in a high BOLD frequency band forecasted positive placebo response suggesting a role of complexity and network-level integration in enabling analgesic responses. Such brain network configurations at baseline may serve as a resource for more effective processing of psychological signals. For instance, efficient information transfer may represent better preparatory resources to facilitate adaptive physiological responses to pain.

To assess whether network configurations facilitate processing of psychological aspects of treatment, we used the Watts- 
Strogatz "small-world" network model to assess network architecture and information flow in the resting brain (Watts and Strogatz, 1998). This model captures how efficiently a given individual's brain transmits information between local and segregated networks (Bullmore and Sporns, 2009). Although this technique is gaining popularity for investigating brain networks that can stratify patients into different neuropsychiatric groups (Bassett and Bullmore, 2009), the functional significance and predictive capacity from a behavioral perspective is relatively unexplored. From a brain-behavior perspective, principles of network organization gleaned from graph-theoretic analysis have shown association with individual variability in working memory (Stevens et al., 2012; Langer et al., 2013) and intellectual ability (van den Heuvel et al., 2009). However, a role of network configurations in facilitating adaptive responses to changes in the environment has so far not been demonstrated. Here, we investigated whether topologic network synchronizations during the baseline period predict responses to psychologically induced analgesia in chronic knee pain patients. To induce analgesia, positive expectations for analgesic effects of acupuncture treatment were experimentally enhanced using verbal suggestion and heat pain conditioning (a model of associative learning). We hypothesized that patients with more efficient network topology show greater analgesic response to psychological conditioning and this relation is invariant to real versus sham acupuncture treatment.

\section{Materials and Methods}

Patient selection criteria. Experiments were conducted with approval from the Massachusetts General Hospital Institution Review Board and with the written consent of each patient. All patients were right-handed and naive to acupuncture treatment to avoid confounds due to previous learning or conditioning effects. Patients were told that the study was an investigation of acupuncture analgesia and were debriefed about the details of the experiment after completion of all study procedures.

Patients suffering with knee osteoarthritis $(\mathrm{OA}$; mean age $=57.9 \pm 7.2$ $\mathrm{SD}, 25$ women) for $\geq 3$ months were recruited. All patients met classification criteria suggested by the American College of Rheumatology for grade 2 or 3 on the Kellgren-Lawrence Scale for radiographically grading knee OA severity (Kellgren and Lawrence, 1957; Gossec et al., 2007; Beattie et al., 2008). In addition, only the patients that had moderate or high levels of clinical pain on most days during the past month $(>15 \mathrm{~d}$ out of 30 , average daily pain of $>3 / 10$ ) in the left and/or right knee were selected.

The exclusion criteria included interventional procedure for knee pain, including corticosteroid injections within 6 months to the knee; intent to undergo surgery during the time of involvement in the study; presence of cardiovascular, pulmonary, neurological or psychiatric or additional pain disorder with severity greater than knee OA pain; pregnancy; and difficulties in reading, speaking, or understanding the English language (Peat et al., 2001). Of the total 67 patients enrolled in the study, 11 were dropped due to discomfort, technical failures, or scheduling issues. Another three patients were dropped after failing screening for illicit drugs and eight more patients were excluded because they did not meet the criteria for stable pain rating in response to calibrated noxious stimuli during the training sessions. The remaining 45 patients completed the study; image data from 2 patients were identified as noisy during preprocessing and these patients were excluded from analyses. One patient was excluded after the incidental discovery of a pre-existing structural brain abnormality. Among the remaining 42 patients, 22 received sham acupuncture and 20 received real acupuncture.

Overview of study design. The study tests the analgesic effects of verbal suggestion and physical conditioning on sham and real acupuncture with a model developed in our previous studies (Kong et al., 2009a,b). Patients participated in three study sessions, where sessions 1 and 2 were used to train patients to rate heat pain using the Gracely Sensory scales (Gracely and Dubner, 1987; Gracely and Kwilosz, 1988) and to determine the appropriate thermal stimulus intensities to be used during the functional magnetic resonance imaging (fMRI) session. The Gracely Sensory Scale is a descriptor differential scale that uses multiple verbal descriptors to anchor pain on a $0-20$ scale. It provides reliable assessments of pain intensity with good test-retest stability and internal consistency (Gracely and Dubner, 1987 Gracely and Kwilosz, 1988). It is recommended for comparison of an individual's response at one point in time to his or her response at a later time or to compare group responses. We have used this scale for investigating placebo responses in several investigations that form the basis for this study (Kong et al., 2005, 2006a, 2008, 2009a,b).

In addition, in session 2, patients underwent an initial conditioning session using verbal suggestion and heat pain conditioning to introduce them to the procedures. Session 3 was the actual test session and was held at a minimum of $3 \mathrm{~d}$ after session 2 . In this session, a resting state fMRI scan was acquired followed by experimental pain conditioning. All experimental procedures and treatments were administered on the right hand.

Thermal pain stimuli and pain assessment. Calibrated thermal pain stimuli were delivered to the right medial aspect of the forearm using a TSA-2001 Thermal Sensory Analyzer with a $3 \times 3 \mathrm{~cm}$ probe (Medoc Advanced Medical Systems). A series of stimuli were applied to one skin site on the volar aspect of the arm using a grid (Kong et al., 2006a). The stimulus was switched to a new site on the grid in random order between series. All stimuli were initiated from a $32^{\circ} \mathrm{C}$ baseline, increased to a target temperature, and presented for $12 \mathrm{~s}$, including a $2.5 \mathrm{~s}$ ramp up and ramp down. Interstimulus interval was jittered randomly to vary between 24 and $30 \mathrm{~s}$. During that interval, patients were presented the Gracely rating scale (Gracely and Dubner, 1987) and were prompted to rate their pain with a button press. Between scans, the thermal probe was moved within the $2 \times 3$ grid to new locations while ensuring that each stimulus series was far enough apart in time ( $\sim 5 \mathrm{~min})$ to avoid sensitization or habituation effects (Kong et al., 2006b; Hashmi and Davis, 2008).

Evoked pain calibration. Session 1 consisted of two phases: calibration phase used for training and stimulus temperature selection and a testing phase. During the calibration phase, participants practiced rating on an ascending series of heat stimuli. Temperatures that elicited subjective intensity ratings in the LOW pain range (5-7 on the $0-20$ Sensory Scale) and HIGH pain range (14-17 on the Sensory Scale) were selected for each participant. Next, the selected HIGH and LOW intensity stimuli were applied in a random sequence to establish reliability of ratings for stimuli presented in an unpredictable order. These sequences were applied on the test and the control side of the arm, and temperatures were adjusted when necessary to ensure that subjective ratings of the temperature established for that participant as HIGH or LOW were in the desired range.

At the end of session 1 and the beginning of session 2, patients were presented with a series of six HIGH stimuli identical to the preconditioning and postconditioning test stimuli to assess how reliably a participant could rate noxious stimuli of identical intensities. Patients had to report approximately equivalent ratings (average pain rating difference $<1.5$ ) between stimulus repetitions and between radial and ulnar sides of their arm to continue with the study. Internal consistency across trials in each condition assessed with Cronbach's $\alpha$ indicated that pain ratings were consistent; $\alpha=0.94$ for all conditions.

Preconditioning pain response. In session 3, patients first rated two repetitions of a series of six HIGH temperature stimuli (total 12 stimuli) applied to two separate skin sites on the radial and ulnar side of their arm (the order of side of arm was randomized between subjects). This testing was conducted to establish a baseline or preconditioning pain response to heat pain.

Verbal conditioning. Patients were psychologically conditioned to believe that acupuncture works only on the side of the arm where the acupuncture needle is inserted. In fact, effects of real acupuncture or sham treatments are generally not lateralized and may affect both sides of the arm. According to the aims of this study, we tested whether verbal suggestion and heat pain conditioning will induce lateralized analgesic effects on the indicated side of the arm. Therefore, after testing for baseline responses, patients were given verbal suggestion with a standardized protocol. Patients were informed that "according to previous literature, 
acupuncture would produce analgesia only on the side of the arm where the acupuncture needle is inserted." To balance the study for laterality affects, half of the patients were psychologically conditioned on the ulnar side and the remaining half on the radial side of the arm. The term "test side" denotes where the patients were conditioned to expect analgesia and "control side" is where patients were conditioned not to expect analgesia. Next, the real acupuncture or sham treatment was applied on the test side.

Acupuncture administration. A licensed acupuncturist (J.K.) performed sham and real acupuncture at LI3 and LI4. Real electroacupuncture involved inserting an acupuncture needle into the skin to a depth of $\sim 1.5 \mathrm{~cm}$ and moving it using Chinese traditional techniques until a sensation of deqi and no sharp pain was evoked. The deqi sensation was quantified with the Massachusetts General Hospital Acupuncture Sensation Scale (MASS; (Kong et al., 2005, 2009b; Zhu et al., 2013). The needles were then connected to an electroacupuncture device, which passed a 2 $\mathrm{Hz}$ current into the skin (OMS Medical Supplies IC-1107; Kong et al., 2005; Zhu et al., 2013). The electric stimulation was gradually increased to the highest level that patients could tolerate without the sensation of sharp pain.

In patients that received sham treatment, Streitberger needles were used as the placebo treatment. The Streitberger needle has been well validated as a sham device (Streitberger and Kleinhenz, 1998; Zhu et al., 2013). The blunt Streitberger needle retracts into its handle when pressed on the skin. The needle was placed on the surface of the skin and connected to a deactivated electroacupuncture device. The procedure (real or sham acupuncture) lasted $25 \mathrm{~min}$.

Heat pain conditioning. This was followed by a physical pain conditioning paradigm; the details of this procedure have been previously published (Kong et al., 2006a). Briefly, a series of heat pain stimuli was applied on the test side where the stimulus intensity was surreptitiously lowered right after the sham (or real) acupuncture treatment to help patients to associate acupuncture with analgesia. On the control side of the arm, patients received temperatures they had associated with HIGH pain from the preceding steps.

Note that the patients were unaware of the stimulus temperatures, psychological maneuvers, and whether they received sham or real acupuncture up to the end of the study. To confirm the effects of the procedures, each patient's expectations were quantified at the beginning of the session and again at the end of the session. Expectations were quantified on a scale from $0-10$, where 0 is "does not work at all (no change)" and 10 is "complete pain relief." Each patient was asked to mark the point on the scale that signified how much he/she expected acupuncture treatment to relieve the heat pain.

Postconditioning pain response. Patients were given only HIGH temperatures on the radial and on the ulnar side of the medial forearm to establish effectiveness of the experimental maneuvers (postconditioning pain response) using identical procedures as those used for establishing the preconditioning pain response.

Questionnaires. Questionnaire data collected in these patients included the Knee Injury and Osteoarthritis Outcome Score (KOOS) for evaluating clinical pain. In addition, we collected demographic information such as age, sex, and behavioral information such as for anxiety levels (State and Trait Anxiety Questionnaire, STAI), depression (Beck Depression Inventory, BDI), and optimism (The Life Orientation Test, LOT) only at the beginning of the study.

Quantification of psychologically conditioned analgesia. The primary endpoint of the procedures was to quantify the analgesia related to psychological conditioning and represents placebo analgesia. Patients were conditioned to believe that the needling procedure would produce analgesia on the test side but not on the control side. To evaluate the effect of psychological conditioning, we first established that percentage decrease in pain ((Postconditioning - Preconditioning)/Preconditioning $\times 100 \%)$ was different between the test and the control sides using a paired $t$ test. Both the preconditioning and postconditioning pain responses were the average of two series of six stimuli. The effect of psychological conditioning was measured as the difference in percentage decrease in pain responses between the test and the control side.
Resting state acquisition. A resting state scan and a structural scan were acquired before commencing the pain testing/conditioning procedures. All brain network analysis was conducted using only the resting state scan and structural data. Data were acquired with a 3 T TIM Trio scanner (Siemens) using a 12-channel phased-array head coil. The functional resting state data were obtained with a gradient-echo echo-planar imaging (EPI) sequence sensitive to BOLD contrast. Structural data were acquired as a high-resolution multi-echo T1-weighted magnetizationprepared gradient-echo image (multi-echo MP-RAGE; van der Kouwe et al., 2008).

While acquiring the resting state scan, patients were instructed to stay awake, keep their eyes open, and remain still. EPI parameters were as follows: $\mathrm{TR}=3000 \mathrm{~ms}, \mathrm{FA}=85^{\circ}, \mathrm{FOV}=216 \mathrm{~mm}, \mathrm{TE}=30 \mathrm{~ms}, 3 \times 3 \times$ $3 \mathrm{~mm}$ voxels, and 47 axial slices collected with interleaved acquisition and no gap between slices. Each resting state scan lasted $6.2 \mathrm{~min}$ (124 time points). The structural scan (multi-echo MP-RAGE; van der Kouwe et al., 2008) parameters were as follows: $\mathrm{TR}=2200 \mathrm{~ms}, \mathrm{TI}=1100 \mathrm{~ms}, \mathrm{TE}=$ $1.54 \mathrm{~ms}$ for echo image $1-7.01 \mathrm{~ms}$ for echo image $4, \mathrm{FA}=7^{\circ}, 1 \times 1 \times 1.3$ $\mathrm{mm}$ voxels, and FOV $=230 \mathrm{~mm}$. The multi-echo MP-RAGE allows increased contrast through weighted averaging of the four derived images.

Data preprocessing. Both FSL and AFNI were used to preprocess data in line with procedures adapted for the 1000 functional connectome project (Biswal et al., 2010). Data were slice time corrected for interleaved acquisitions using Fourier interpolation, motion corrected using least-squares alignment of each volume to the eighth image using Fourier interpolation, despiked of extreme time series outliers using a continuous transformation function, temporal bandpass filtered between $0.009-0.2 \mathrm{~Hz}$ using Fourier transformation, and filtered to remove linear and quadratic trends using AFNI. In addition, FSL was used for spatially smoothing the images (Gaussian kernel full-width at half-maximum $=6 \mathrm{~mm}$ ), and for normalizing mean-based intensity by the same factor $(10,000)$. Next, nine nuisance signals (global mean, white matter (WM), CSF signals, and six motion parameters) were regressed out. The global signal regressor was generated from a whole-brain mask by averaging across the time series in all voxels in the mask. WM and CSF covariates were generated by first segmenting each individual's high-resolution structural image (using FAST in FSL), and second, thresholding the segmented WM and CSF images to ensure $80 \%$ tissue type probability. Finally, the BOLD time series was extracted from every voxel within a given mask and averaged to generate the WM and CSF nuisance signal. The six motion parameters generated in the FSL-based motion-correction step were used to correct for motion. These six vectors included rotational movement around three axes (pitch, yaw, and roll) and movement in each of the three cardinal directions $(X, Y$, and $Z$ ). All of these steps were conducted in native functional space.

For registration, FLIRT and FNIRT were used for transformations from native functional and structural space to the Montreal Neurological Institute MNI152 template with $2 \times 2 \times 2 \mathrm{~mm}$ resolution. First, the high-resolution structural image was registered to the MNI152 $2 \mathrm{~mm}$ template with a 12 degree-of-freedom linear affine transformation. The transformation was further refined using FNIRT nonlinear registration. Next, each participant's functional data were registered to their highresolution structural image using a linear transformation with 6 degrees of freedom. The structural-to-standard nonlinear transformation matrix was used to register the functional volume to MNI152 standard space.

Brain parcellation and time course extraction. To define nodes, we used the Harvard Oxford Atlas to parcellate the brain. The atlas was modified to separate the regions of interest (ROIs) that spanned both hemispheres into separate ROIs for the right and left hemispheres. Regions above the paramedial sulcus were labeled as dorsal medial prefrontal cortex (PFC) and this region was further separated into an anterior (dMPFCa) and a posterior (dMPFCp) region (Fig. 2A, middle bottom). The region occupying the superior rostral sulcus below the paramedical sulcus was demarcated as medial prefrontal (MPFC). The inferior rostral sulcus was demarcated as ventral medial prefrontal cortex (vMPFC). The lateral aspect of the frontal pole (FP) was subdivided into a superior region labeled as FP (Fig. 2A, right top) and an inferior region labeled as orbitofrontal pole (OFP; Fig. $2 A$, right top) where the two regions are approx- 
imately above and below the frontomarginal sulcus, respectively. The single ROI for the cingulate cortex was parcellated into the seven subregions identified by Beckmann et al. (2009) derived from clustering of functional and structural (tractography-based) connections of this region with the rest of the brain. The regions are shown in Figure 2 (middle bottom). The remaining regions were retained as originally present in the Harvard Oxford Atlas. The list of these regions and their coordinates are given in Table 2. The 125 regions are designated as nodes for constructing the graph. The BOLD time series were extracted from each voxel within each node and averaged resulting in 125 time series in each patient. These time series were used for observing network properties with graph analysis (Fig. 2B).

Graph construction. The networks were constructed and network measures were assessed using the Brain Connectivity Toolbox created by Rubinov and Sporns, 2010 (https://sites.google.com/site/bctnet/). All formulae used for calculating network measures are provided in their paper (Rubinov and Sporns, 2010) and are available on the Toolbox website. For each patient, the connectivity graph was constructed by creating a $125 \times 125$ correlation matrix. The network was binarized by thresholding the correlation matrix at values of $T$ ranging from 0.1 to 0.5 in 0.05 increments $(T=0.1,0.15,0.2,0.25,0.3,0.35,0.4,0.45,0.5)$. Thus if two regions were connected at a value below $T$, the connection was set equal to zero. All remaining cells were set equal to one. Low values of $T$ carry the risk of overestimation due to noisy, weak, or physiologically insignificant connections. High thresholds, on the other hand, can fragment the networks into a collection of smaller networks that lead to overlooking correlations of functional importance and misrepresenting the graph structure. There is no definitive method for selecting optimal thresholds; hence it is customary to use a range of plausible thresholds and visualize the results (Stevens et al., 2012).

We measured reachability at the tested thresholds to detect fragmented networks. Average reachability of the network is a measure of the proportion of nodes that are connected by any path, regardless of the path length (i.e., the number of intervening nodes that must be traversed to reach one node from the other). A low reachability score implies a fragmented graph where some nodes are completely disconnected from all others in the network (Stevens et al., 2012). The average reachability of the network was exactly 1 for values of $T=0.1,0.15,0.2,0.25,0.3$, and 0.35 , which indicates that the graphs were completely connected. At higher thresholds, a few nodes were disconnected from the graph (1 node for most networks and in some cases $2-5$ nodes especially at $T=0.5$ ) and hence removed before computing metrics. This shows that the graphs were fully connected for most thresholds tested.

Thresholding at fixed values of $T$ may yield networks that vary in number of edges between subjects. First, as a control, we measured mean degree of every network. This metric calculates the number of connections of a node averaged over all nodes and is thus a measure of mean connectivity. In addition, we tested whether the main finding could be reproduced with network density fixed in each subject (van Wijk et al., 2010). For this we calculated the network threshold that was defined as the total number of edges in a network divided by the maximum possible number of edges, which results in cortical networks that have the same number of connections in each network. Each correlation matrix was thresholded over a wide range of link densities ranging from 0.1 to 0.5 at 0.1 increments.

Graph-theoretic metrics of network topology. We used graph-theoretic metrics that are used for assessing network topology associated with small-world organization in the brain (Bullmore and Sporns, 2009; Rubinov and Sporns, 2010). Small-world organization is depicted as clusters of closely linked nodes where each cluster is connected with other clusters with a few links between hubs within the cluster. This type of organization reduces cost while allowing effective information sharing between nodes. Small-world organization uses clustering coefficients (Ccoef) and characteristic path length (Plength) for identifying the topological structure of a network. The main objective of this investigation was to assess whether individual variability in network topology and information transfer predicts psychologically induced analgesia. For this, we selected four measures that reflect network topology and efficiency of information transfer at the local level (Ccoef, local efficiency) and global level (Plength, global efficiency).

Clustering coefficients, as the name suggests, measures the amount of clustering in the network and is measured as the average proportion of connectivity between each node's nearest neighbors. Clustering coefficients is a measure of connectivity between neighbors or cliquiness within a large-scale network. This property is also used for measuring small-world organization by comparing the amount of clustering observed in the network to a random network. Characteristic path length measures the average number of links that must be traversed to connect any two nodes in the network. "Network efficiency" is a measure of the network's capacity for parallel information transfer between nodes via multiple series of edges. Global efficiency measures how efficiently information can be transferred among nodes in the entire network (Latora and Marchiori, 2001) and is computed as the inverse of the characteristic path length. Local efficiency reflects how effectively information is transferred in a local network (Ginestet et al., 2011) and is calculated as the average of all the subgraph's global-efficiencies where each subgraph is composed by the neighbors of a given node.

Statistics. Each of the selected metrics was computed for each node and averaged over all nodes in the brain network of every patient for different values of $T$. This mean value calculated in each patient was correlated with his/her respective analgesic response (percentage pain decrease) using a Pearson correlation. The metrics that showed a relation after correcting for multiple comparisons at a false discovery rate (FDR) of 0.05 were considered to be significant.

Since the mean clustering coefficient showed a significant prediction of analgesia, we further assessed the predictive strength of the relationship by plotting prediction intervals in linear regressions measured in networks constructed at $T=0.25$ and confirmed the relationship with robust regression. Prediction intervals were plotted to specify at what probability a random variable selected from a future observation will lie within a certain interval based on the current observation. For example, if we collect a sample of observations and calculate a $95 \%$ prediction interval based on that sample, there is a $95 \%$ probability that a future observation will be contained within the prediction interval.

Due to a lack of a clear binarization between responders and nonresponders, the magnitude of experimental modulation in pain was investigated mainly as a continuous variable. However, to visually explore differences in network clustering, patients were separated into two equal groups (responders and nonresponders) based on a median split in percentage pain decrease (median $=-8.5$ ).

To identify the underlying nodes that most robustly correlated with the analgesic response, clustering coefficient and centrality measures (between-ness and degree) of each individual node were correlated with conditioned analgesia at a network threshold of $T=0.25$ and the results were corrected for multiple comparisons with FDR and regions were displayed using BrainNet Viewer (Xia et al., 2013).

\section{Results}

\section{Psychologically induced analgesia is invariant to real versus sham acupuncture}

The psychological maneuvers used in the study (verbal and physical conditioning) had a significant influence on evoked pain responses observed in sham acupuncture- and real acupuncturetreated patients. In the sham group, pain decreased significantly more $\left(T_{(1,21)}=3.14, p=0.0049\right.$; paired $t$ test $)$ from preconditioning-evoked pain (MEAN $=13.43 \pm 0.50 \mathrm{SEM})$ to postconditioning-evoked pain $(\mathrm{MEAN}=12.23 \pm 0.69 \mathrm{SEM})$ in the side of the arm where patients were psychologically conditioned to expect analgesia (test side). In comparison, there was no significant change in pain $\left(T_{(1,21)}=-1.81, p=0.085\right.$; paired $t$ test) in the control side between preconditioning $(\mathrm{MEAN}=$ $13.6 \pm 0.49 \mathrm{SEM})$ and postconditioning $(\mathrm{MEAN}=14.03 \pm 0.5$ SEM). The percentage decrease in pain that occurred in the test side $(\mathrm{MEAN}=-9.65 \pm 3.17 \mathrm{SEM})$ was thus significantly greater 
$\left(T_{(1,21)}=-3.41, p=0.0026\right)$ than in the control side $(\mathrm{MEAN}=4.23 \pm 2.12 \mathrm{SEM})$ as shown in Figure $1 A$.

A similar pattern was observed in the acupuncture-treated patients where the preconditioning-evoked pain (MEAN = $12.74 \pm 0.47$ SEM) was significantly higher $\left(T_{(1,19)}=3.35, p=0.0033\right.$; paired $t$ test $)$ than the postconditioning pain (MEAN = $11.5 \pm 0.53 \mathrm{SEM})$ in the test side. The control side showed no significant change $\left(T_{(1,21)}=-0.62, p=0.53\right.$; paired $t$ test $)$ in evoked pain between preconditioningevoked $(\mathrm{MEAN}=12.5 \pm 0.46 \mathrm{SEM})$ and postconditioning-evoked pain (MEAN = $12.95 \pm 0.42$ SEM). The percentage change in pain that occurred in the test side $(\mathrm{MEAN}=-9.35 \pm 2.84 \mathrm{SEM})$ was thus significantly greater $\left(T_{(1,19)}=-2.98, p=\right.$ $0.0077)$ than in the control side (MEAN $=3.87 \pm 2.4$ SEM; Fig. $1 A)$. The final outcome of psychological conditioning on evoked pain (percentage decrease in test side - control side) was therefore not significantly different $\left(T_{(1,40)}=0.11, p=0.91\right)$ between the sham $(\mathrm{MEAN}=-13.89 \pm 4.1 \mathrm{SEM})$ and acupuncture $(\mathrm{MEAN}=$ $-13.23 \pm 4.44$ SEM) groups (Fig. $1 B$ ). Since we had verified that the psychologically induced analgesia was invariant to sham or real acupuncture, the results were pooled from both groups for subsequent analyses.

Expectations for pain relief were significantly correlated with percentage analgesia before $\left(r=-0.50,{ }^{\star} p=0.001\right)$ and after the imaging session $\left(r=-0.41,{ }^{\star} p=0.008\right)$. Note that there was no correlation between percentage analgesia and age, KOOS pain, disease duration, anxiety, or depression $(p>0.05)$ measured at baseline (i.e., before the imaging session; Table 1).

The psychological conditioning did not work equally well in all patients. The marked interindividual variability in the analgesic response is shown in Figure $1 A$ and $C$. The percentage analgesia values representing each individual's response to psychological conditioning were used for investigating brain network properties that underlie individual differences in psychologically induced analgesia.

\section{Association between graph-theoretic metrics and psychologically induced analgesia}

We used a structured approach and investigated local and global graph-theoretic measures (Fig. 2; Table 2) that explain individual differences in levels of psychologically induced analgesia. Thus, on investigating whether local metrics (clustering coefficients and local efficiency) or global metrics (path length and global efficiency) predicted analgesic response, we observed a significant and consistent relation with local network measures. Clustering coefficients correlated with subsequent placebo analgesia measured at most network thresholds (Fig. 3A): $T=0.1(r=$ $\left.-0.40,{ }^{\star} p=0.008\right), T=0.15\left(r=-0.44,{ }^{\star} p=0.003\right), 0.2(r=$ $\left.-0.45,{ }^{\star} p=0.003\right), 0.25\left(r=-0.46,{ }^{\star} p=0.002\right), 0.3(r=-0.43$, $\left.{ }^{\star} p=0.004\right), 0.35\left(r=-0.38,{ }^{\star} p=0.012\right), 0.4\left(r=-0.37,{ }^{\star} p=\right.$ $0.018), 0.45\left(r=-0.40,{ }^{\star} p=0.010\right)$, and $0.5(r=-0.30, p=$ 0.054 ; overall FDR corrected value $q=0.018$ ). Similarly, local efficiency also showed a relationship with placebo analgesia: $T=$ $0.1\left(r=-0.43,{ }^{\star} p=0.0052\right), T=0.15\left(r=-0.45,{ }^{\star} p=0.0028\right)$, $0.2\left(r=-0.44,{ }^{\star} p=0.0038\right), 0.25\left(r=-0.42,{ }^{\star} p=0.0058\right), 0.3$ $\left(r=-0.41,{ }^{\star} p=0.0071\right), 0.35\left(r=-0.35,{ }^{*} p=0.021\right), 0.4(r=$ $\left.-0.37,{ }^{\star} p=0.020\right), 0.45\left(r=-0.39,{ }^{\star} p=0.011\right)$, and $0.5(r=$
Table 1. Relation between psychologically induced analgesia, demographic, and behavioral data

\begin{tabular}{lccl}
\hline Parameters & Mean & $R$ value & $p$ value \\
\hline Age (years) & $57.9 \pm 7.2$ & 0.25 & 0.1 \\
Female sex, $\mathrm{n}$ & 25 & $-0.31^{*}$ & 0.047 \\
Duration (years) & $4.6 \pm 3.2$ & 0.21 & 0.28 \\
KOOS pain intensity & $58.2 \pm 15.6$ & 0.046 & 0.77 \\
STAl & $25.6 \pm 6.0$ & 0.07 & 0.66 \\
BDI & $5.2 \pm 6.8$ & 0.032 & 0.84 \\
LOT & $23.3 \pm 5.17$ & -0.004 & 0.98 \\
\hline
\end{tabular}

Age and behavioral data showed no significant relationship with placebo analgesia. Patient sex showed a marginally significant relation suggesting greater placebo analgesia in women.

$-0.33,{ }^{*} p=0.033$; over all FDR corrected value $\left.q=0.023\right)$. Some nodes were disconnected at $T=0.4,0.45$, and 0.5 , and therefore removed before calculating the metrics. However, the significant relation was consistent across the range of thresholds. Due to the similarity and overlap between clustering coefficients and local efficiency, subsequent analysis only used clustering coefficient to represent local connectivity. Note that characteristic path length and global efficiency were not significantly associated with analgesic response after FDR correction $(q>0.05$, Fig. $3 A)$. The identified relationship was significant even after the multiple comparisons for all five metrics and all thresholds were combined in a single vector and corrected with FDR (main effect $q=0.021$ ). We also confirmed that the significant findings were not due the variability in baseline preconditioning pain $(p>0.05)$. In addition, mean degree was not significantly correlated with placebo analgesia indicating that local network topology and not mean global connectivity predicted pain modulation (Fig. 3A).

On separating the patients into responders and nonresponders using a median split of the percentage decrease (median $=-8.5$ ) as an arbitrary classification of patients into two equal groups (Fig. 3B), the responders visibly showed greater network clustering than in nonresponders. To calculate the strength of the prediction, the relation between percentage decrease in pain and clustering coefficient measured at $T=0.25$ was plotted with prediction intervals and tested with a linear regression (Fig. $3 C$ ). There was a robust relationship between clustering coefficients and psychologically induced analgesia $(\beta=-0.46$, $\left.{ }^{\star} p=0.002\right)$. The coefficients remained significant when the relation was further confirmed with robust regression. There was no effect of treatment type on the relationship between clustering coefficients and placebo analgesia $(\beta=0.01, p=0.99)$. 


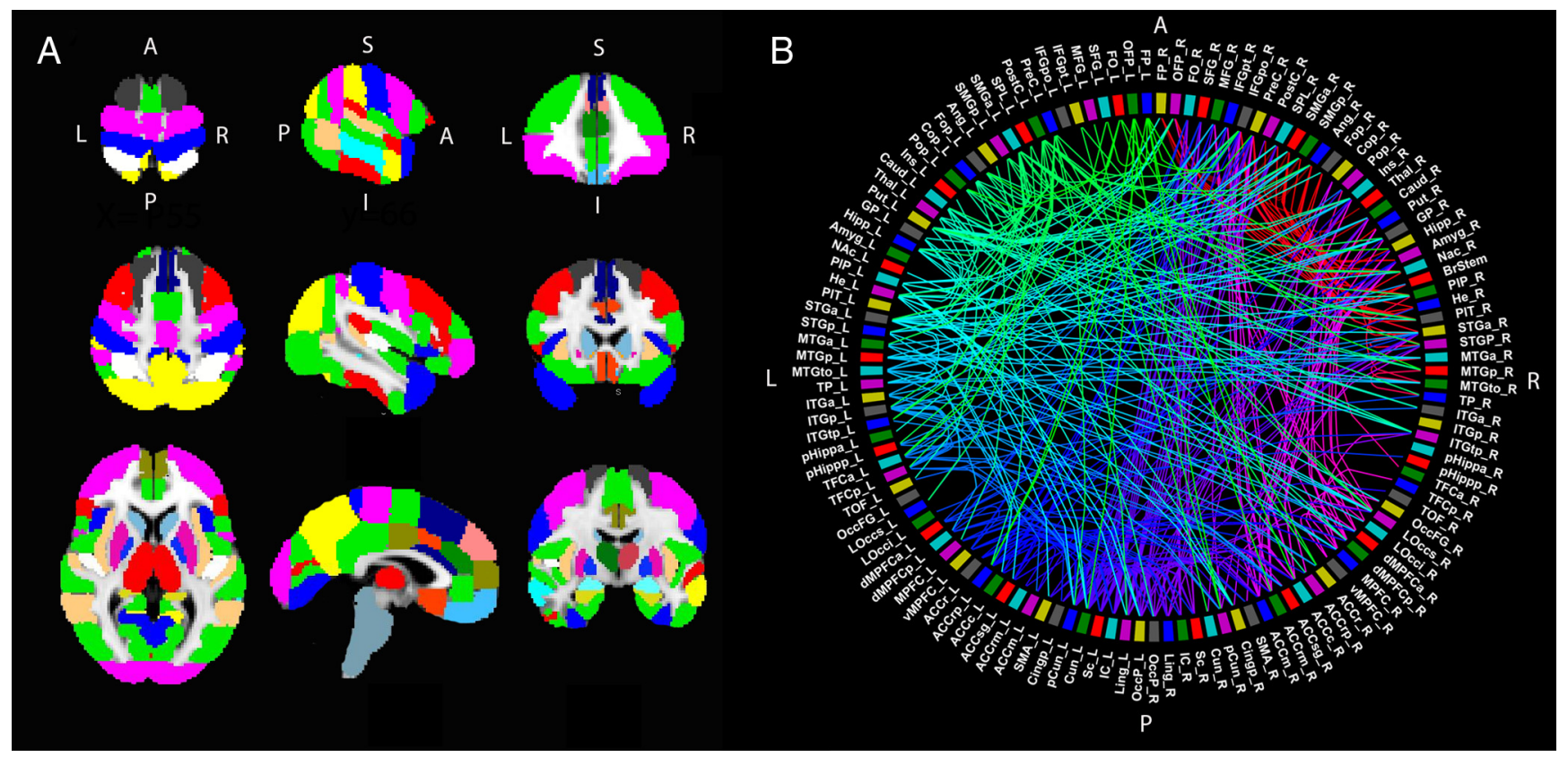

Figure 2. Parcellation scheme used for measuring network properties in the brain of resting $0 \mathrm{~A}$ patients. $A$, The network was constructed from 125 regions based on the Harvard $0 x f$ ord Atlas (see Table 2 for list of coordinates). $\boldsymbol{B}$, Circle diagram showing local connectivity near the perimeter and long distance connections between nodes shown in randomly colored edges (mean of $n=42$ displayed at $T=0.6)$. The right $(\mathrm{R})$ and left $(\mathrm{L})$ sides of the circle represent the two brain hemispheres and the ordering of the nodes from top to bottom is organized based on their respective positions on the brain from anterior (A) to posterior (P). S, superior; I, inferior. For abbreviations in $\boldsymbol{B}$, see Table 2 .

In addition, partial correlation coefficients were significant for most values of $T: T=0.1\left(r=-0.40,{ }^{\star} p=0.008\right), 0.15(r=$ $\left.-0.44,{ }^{\star} p=0.003\right), 0.2\left(r=-0.44,{ }^{\star} p=0.003\right), 0.25(r=-0.46$, $\left.{ }^{\star} p=0.002\right), 0.3\left(r=-0.43,{ }^{*} p=0.004\right), 0.35\left(r=-0.38,{ }^{*} p=\right.$ $0.012), 0.4\left(r=-0.36,{ }^{\star} p=0.012\right)$, and $0.45\left(r=-0.40,{ }^{\star} p=\right.$ 0.001 ) after correction for treatment group (sham vs real acupuncture $)$ and the relation was significant $(q=0.018)$ after FDR correction for multiple comparisons.

To determine whether the findings were dependent on network size, we measured the relation between clustering coefficients and percentage pain decrease by using a constant link density in all patients. There was a significant correlation between analgesia and clustering coefficients for link density adjusted at $0.1\left(r=-0.37,{ }^{\star} p=0.014\right), 0.2\left(r=-0.41,{ }^{\star} p=0.0065\right), 0.3$ $\left(r=-0.44,{ }^{\star} p=0.0033\right), 0.4\left(r=-0.44,{ }^{\star} p=0.0034\right)$, and 0.5 $\left(r=-0.46,{ }^{\star} p=0.002\right)$. Network clustering coefficients adjusted at 0.25 link density shown in Figure $3 D$ showed a significant correlation with analgesia $\left(r=0.44,{ }^{*} p=0.003\right)$ with no significant interaction for sham versus real acupuncture $(p>0.05)$. Since the main finding was not affected by the thresholding method, to avoid redundancy, the remaining analyses were conducted using fixed thresholds.

\section{Effect of age and behavioral measures}

There was no significant relation between age and percentage decrease in pain $(r=0.26, p=0.10)$. Moreover, we did not find any significant relation of age with clustering coefficients $(p>$ $0.05)$. Hence the relation between percentage decrease in pain and clustering coefficients was not affected when effects of age were regressed out with a partial correlation and remained significant for the same values of $T$ that showed significance before correction. Clustering coefficients showed no significant relationship with knee pain (KOOS pain), anxiety (STAI), or depression (BDI) scores measured before the imaging session when tested at all network thresholds $(p>0.05)$ measured before the imaging procedures. In addition, the patient's expectations for pain relief did not correlate with clustering coefficients either before or after session 2 (training session) or at any time point during session 3 (imaging and test session) $(p>0.05)$.

\section{Sex effects}

There was a marginally significant $\left(r=0.31,{ }^{\star} p=0.047\right)$ correlation between sex and percentage decrease in pain indicating that more women responded to the conditioning effects (Fig. 4A, Table 1). Moreover, women showed significantly higher clustering coefficients than men (FDR corrected $p=0.033$ ) for values of $T=0.15,0.2,0.25,0.3,0.35$, and 0.4 (Fig. $4 B, C$ ). On the other hand, clustering coefficients were coupled with analgesic response in men, as well as in women and partial correlations confirmed that the main findings were not driven by a sex effect so that the relationship between clustering coefficient and analgesia remained significant after correcting for sex for $T=0.1(r=$ $\left.-0.34,{ }^{\star} p=0.028\right), 0.15\left(r=-0.38,{ }^{\star} p=0.014\right), 0.2(r=-0.38$, $\left.{ }^{\star} p=0.014\right), 0.25\left(r=-0.4,{ }^{\star} p=0.010\right), 0.3\left(r=-0.37,{ }^{\star} p=\right.$ $0.018), 0.35\left(r=-0.31,{ }^{\star} p=0.045\right)$, and $0.45\left(r=0.33,{ }^{\star} p=\right.$ 0.033 ; FDR corrected $q=0.033$ ). Thus, the overall coupling between pain modulation and graph metrics was applicable to both men and women.

A multivariate ANOVA showed that women were not behaviorally different from men in baseline anxiety, depression, knee pain, or optimism $(p>0.05)$. However, positive expectations toward pain relief were significantly greater in women before the imaging session commenced $\left(F_{(1,41)}=4.9, p=0.034\right)$ and also at the end of the imaging session $\left(F_{(1,41)}=4.4, p=0.043\right)$. In addition, we observed no sex difference in head motion (mean absolute displacement $\left(F_{(1,41)}=1.6, p=0.21\right)$ and there was no association between mean absolute displacement and clustering coefficients at any value of $T(p>0.05)$. Furthermore, regional analysis for sex differences in clustering coefficients (tested at $T=$ 
$0.25)$ was not significant after correction for multiple comparisons $(q>0.05)$.

\section{Brain regions with topological properties associated with psychological conditioning}

Among the regions listed in Table 2, some regions showed clustering coefficients that correlated with percentage pain decrease after FDR correction $(T=0.25$; Fig. $5 A)$. In particular, regions involved in cognitive modulation of pain and emotion (for example, dorsal parts of lateral and medial PFC, and anterior cingulate), motivational brain circuitry (medial PFC, nucleus accumbens, putamen, and globus pallidus), memory (middle temporal regions, posterior cingulate, and hippocampus) as well as visual circuitry were closely linked with experimentally induced analgesia. Correlation values for individual regions are shown in Table 3. On testing whether these regions represent the circuitry driving the relationship, clustering coefficients were computed after removing all 21 regions that had survived correction (Fig. 5A, Table 3). We found that global clustering coefficients computed from the remaining 113 regions significantly correlated with analgesia $(r=0.32$, $\left.{ }^{\star} p=0.036\right)$. Thus, a few discrete regions were more closely linked with mediating this effect, but they were not critical in driving the relation of analgesia. This suggests that large-scale network topology predicted the relationship with analgesia and was not dependent on a few regions (Fig. 5A, Table 3).

The nodal degree (a hub characteristic) also showed a significant relation with percentage pain decrease in several nodes in the medial PFC (left and right dorsal, medial and ventral), left frontal and orbitofrontal pole and left hippocampus. A higher nodal degree in these regions predicted greater pain modulation (Fig. 5B). Between-ness centrality, which is an alternative metric for computing hub-ness showed no distinct regions that correlated significantly with analgesia after FDR correction $(q>0.05)$.

The vMPFC is centrally involved in pain and emotion regulation (Roy et al., 2012), especially in situations that require building of associations between cognitive processes and somatic cues (Damasio, 1996; Lim et al., 2013; Winecoff et al., 2013). We tested the functional connectivity of the right vMPFC known for its role in negative affect (Hilz et al., 2006) with other nodes to understand its role in adaptive pain responses to psychological cues. There was a significant relationship between pain modulation and the vMPFC functional connections with the dorsolateral PFC (dlPFC; middle frontal gyrus, MFG), anterior cingulate, thalamus, hippocampus, parahippocampal gyrus, and middle temporal gyrus (Table 3) after FDR correction.

A

B puncture. ${ }^{*} p<0.05$.

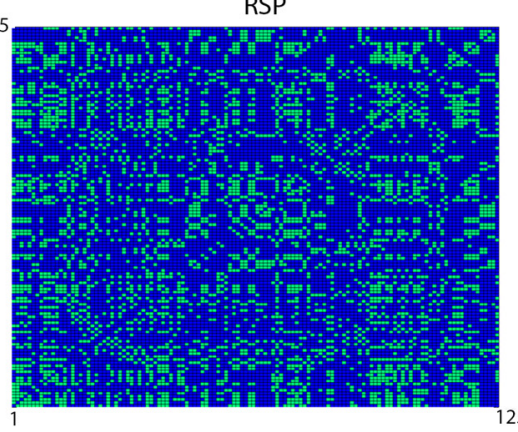

corr < Small world metric. \% decrease in pain $>$
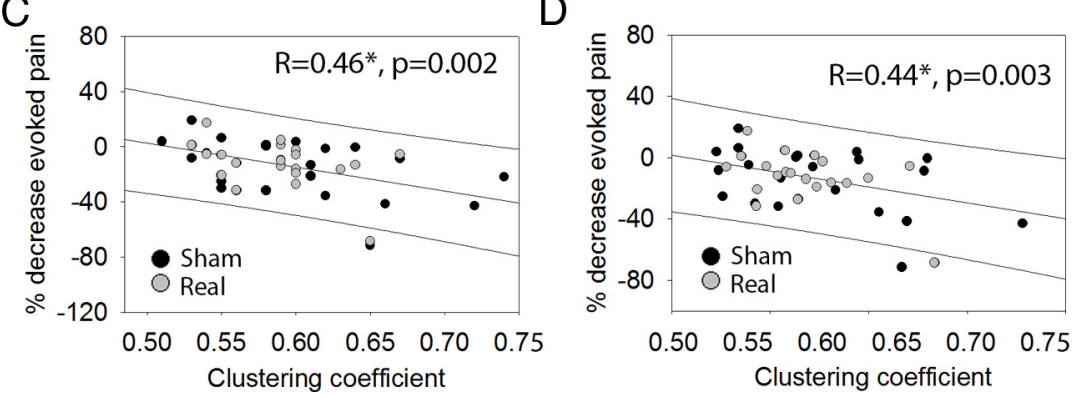

Figure 3. Network clustering in resting brain of OA patients predicts subsequent psychologically induced analgesia. $A$, Mean clustering coefficients (CCoef) and local efficiency (LocEff) significantly correlates with percentage pain decrease at different network thresholds (FDR corrected for multiple comparisons $q<0.05$ ). Mean characteristic path length (Plength) and mean global efficiency (GloEff) did not show a significant correlation after correction. $\boldsymbol{B}$, Correlation matrix $(n \times n)$ for the 125 brain regions (binarized at $T=0.25$ ) visually shows greater clustering in brain networks of responders (RSP) than in nonresponders (NRSP). The two patient groups were derived based on a median split of analgesic response to psychological conditioning (percentage decrease in pain). The ordering of the 125 regions is the same as in Table 2. C, Regression plots with prediction intervals setween mean clustering coefficients (measured at $T=0.25$ ) and analgesia was not affected by the when networks were thresholded using a constant link density of 0.25 with no significant interaction for sham versus real acu-

\section{Discussion}

Placebo conditioning is an experimental model for studying effects of psychological factors such as positive expectations and previous learning in mediating analgesia. Consistent with our previous studies that used a similar model in healthy subjects (Kong et al., 2009a,b), we found that psychological factors are able to induce experimental analgesia (as indicated by reduction in evoked pain) in chronic knee OA pain patients (Price et al., 2007). As previously observed in healthy subjects, effects of psychological conditioning were comparable for real and sham acupuncture. We find that this psychobiological pain modulation can be forecasted by how efficiently information is shared within 
A

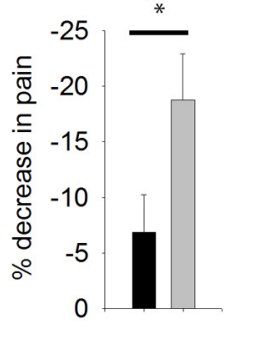

Men Women
B

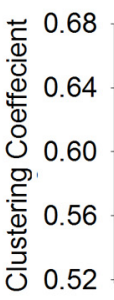

0.52

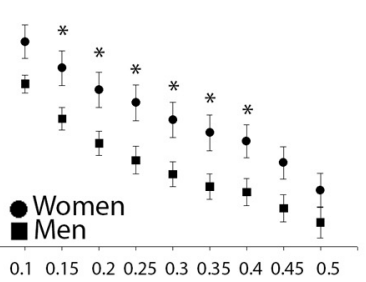

Network threshold (T)

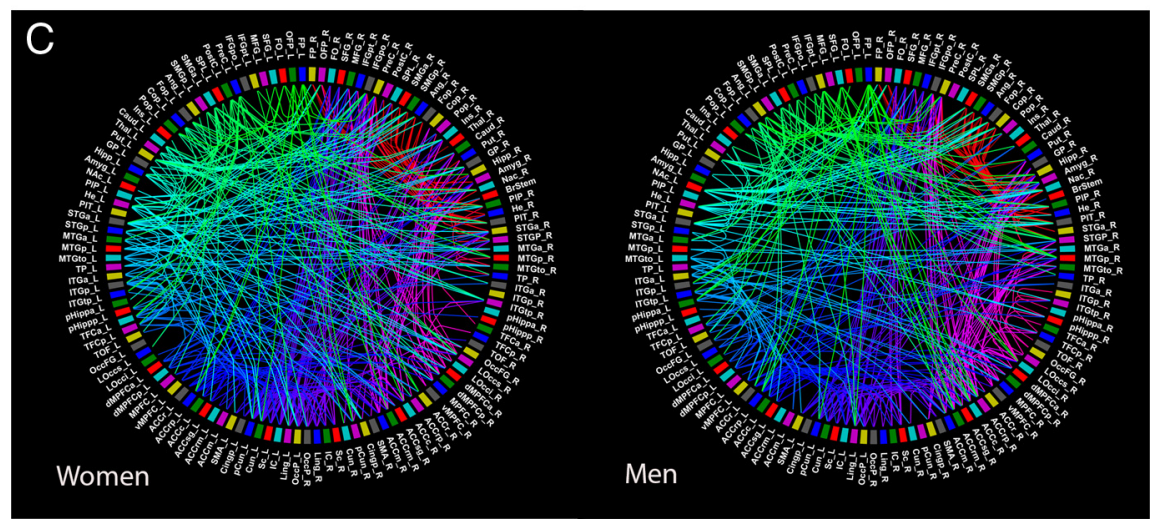

Figure 4. Sex difference in brain network properties and analgesic response to psychological conditioning. $\boldsymbol{A}$, Psychologically induced analgesia was significantly greater in women than in men. $\boldsymbol{B}$, Women suffering with 0 A showed significantly greater clustering coefficients than men at most network thresholds. C, Circle diagrams showing network connectivity in women and men at $T=0.6,{ }^{*} p<0.05$. Error bars indicate \pm SEM. For abbreviations, see Table 2.

local networks in the brain. We provide evidence that network topology of a resting brain prefaces the extent of pain modulation that occurs after subsequent experimental procedures. This is a first demonstration that topological network property predicts adaptive response to environmental cues.

Brain imaging studies have shown psychobiological correlates in the brain that process cognitive inputs associated with placebo analgesia (Kong et al., 2006a; Meissner et al., 2011). Neuropharmacologic studies in animals and humans implicate the endogenous opiate system and dopaminergic-reward circuitry in placebo analgesia (Benedetti et al., 2005; Zubieta et al., 2005, 2006; Colloca et al., 2013). The level of engagement of these systems varies in subjects and this individual variance is directly linked with the extent of placebo-induced analgesia (Zubieta et al., 2005; Scott et al., 2007, 2008). In addition, cognitive networks activated in relation to placebo analgesia were shown to interact with pain and affect modulating systems of the brain (Wager et al., 2011; Hashmi et al., 2012; Kong et al., 2013). It is now being discovered that the regions activated by placebo task after treatment also show baseline activation signals before the placebo task (Watson et al., 2009; Wager et al., 2011; Kong et al., 2013). For instance, baseline brain activations predict analgesic responses to placebo conditioning in healthy subjects (Wager et al., 2011). Furthermore, functional connectivity between task localized regions observed in a pretreatment scan was predictive of subsequent analgesia to placebo treatment in a clinical trial in chronic back pain patients (Hashmi et al., 2012). More revealing were findings that lateral frontal regions, which are invariably linked with processing cognitive aspects of placebo treatments (Craggs et al., 2007; Petrovic et al., 2010; Colloca et al., 2013), showed greater power in the high-frequency band $(0.12-0.2 \mathrm{~Hz})$ and synchronization with a set of distinct regions widespread over the

brain in responders relative to nonresponders. In place of task localization, here we measured the architecture of functional connections integrated over the entire brain to see if it facilitates psychologically induced analgesia. Local network architecture such as local clustering and local efficiency were correlated with subsequent response to expectancy enhancing cues associated with a treatment. A higher clustering coefficient is considered to measure cliquiness within a network and captures the network's resilience against random node damage and thus estimates the robustness of a network (Stam and Reijneveld, 2007). From an information theory perspective, greater clustering is associated with lower cost of information transfer and the capacity to transfer information locally in a network (Bullmore and Sporns, 2009). The reasons for individual variance in these network properties and associated analgesic response are not clear and require further investigation. One proximate cause for these differences could be short term, such as participation in the experiment or the initial training. Another plausible explanation is long term learning effects of previous treatment history and/or the individual's genetic makeup.

The extent to which network architecture can influence behavior or facilitate learning has not been clearly investigated. Here we report that local networks that are typically associated with memory, learning, and conditioning show better information processing in relation with stronger analgesic response to conditioning. The regions with topological characteristics conducive for conditioned analgesia were also remarkably similar to regions previously shown to be mobilized by placebo tasks in positron emission tomography and fMRI investigations (Petrovic et al., 2005, 2010; Kong et al., 2006a; Craggs et al., 2007, 2008; Eippert et al., 2009). Thus, the analgesic response was associated with greater local connectivity of regions involved in cognitive modulation of pain (dorsal PFC; (Benedetti, 2008; Price et al., 2008; Hashmi et al., 2012) and motivation and learning (nucleus accumbens, medial PFC, hippocampus, temporal cortices, and posterior cingulate; Brown and Aggleton, 2001; Glahn et al., 2005; Baliki et al., 2010; Hashmi et al., 2013). Among these regions, the nodal degree of prefrontal nodes and the hippocampus showed higher global connectivity in relation with greater placebo analgesia suggesting that these regions are important hubs in facilitating placebo analgesia. In addition, stronger vMPFC connections with limbic and memory circuitry on one hand and executive control regions such as the dIPFC (MFG) and dMPFC on the other, were observed in relation to placebo analgesia. This connectivity pattern is an important mechanism implicated in integrating contextual information with emotional or painful processes such as building associations between cognitive processes and somatic cues (Damasio, 1996; Roy et al., 2012). Here we observe how these functions may be important in mediating placebo conditioning and illustrate how 
Table 2. Parcellation scheme used for defining brain nodes

\begin{tabular}{|c|c|}
\hline & Name \\
\hline 1 & Frontal pole \\
\hline 2 & Orbitofrontal pole \\
\hline 3 & Frontal orbital cortex \\
\hline 4 & Superior frontal gyrus \\
\hline 5 & Middle frontal gyrus \\
\hline 6 & Inferior frontal gyrus, pars triangularis \\
\hline 7 & Inferior frontal gyrus, pars opercularis \\
\hline 8 & Precentral gyrus \\
\hline 9 & Postcentral gyrus \\
\hline 10 & Superior parietal lobule \\
\hline 11 & Supramarginal gyrus, anterior division \\
\hline 12 & Supramarginal gyrus, posterior division \\
\hline 13 & Angular gyrus \\
\hline 14 & Frontal operculum cortex \\
\hline 15 & Central opercular cortex \\
\hline 16 & Parietal operculum cortex \\
\hline 17 & Insular cortex left \\
\hline 18 & Caudate \\
\hline 19 & Thalamus \\
\hline 20 & Putamen \\
\hline 21 & Globus pallidus \\
\hline 22 & Hippocampus \\
\hline 23 & Amygdala \\
\hline 24 & Nucleus accumbens \\
\hline 25 & Planum polare \\
\hline 26 & Heschl's gyrus (includes $\mathrm{H} 1$ and $\mathrm{H} 2$ ) \\
\hline 27 & Planum temporale \\
\hline 28 & Superior temporal gyrus, anterior division \\
\hline 29 & Superior temporal gyrus, posterior division \\
\hline 30 & Middle temporal gyrus, anterior division \\
\hline 31 & Middle temporal gyrus, posterior division \\
\hline 32 & Middle temporal gyrus, temporo-occipital \\
\hline 33 & Temporal pole \\
\hline 34 & Inferior temporal gyrus, anterior division \\
\hline 35 & Inferior temporal gyrus, posterior division \\
\hline 36 & Inferior temporal gyrus, temporo-occipital \\
\hline 37 & Parahippocampal gyrus, anterior division b \\
\hline 38 & Parahippocampal gyrus, posterior division \\
\hline 39 & Temporal fusiform cortex, anterior division \\
\hline 40 & Temporal fusiform cortex, posterior divisior \\
\hline 41 & Temporal occipital fusiform cortex \\
\hline 42 & Occipital fusiform gyrus \\
\hline 43 & Lateral occipital cortex, superior division \\
\hline 44 & Lateral occipital cortex, inferior division \\
\hline 45 & Dorsal medial prefrontal cortex, anterior \\
\hline 46 & Dorsal medial prefrontal cortex, posterior \\
\hline 47 & Medial prefrontal cortex \\
\hline 48 & Ventral medial prefrontal cortex \\
\hline 49 & Rostral anterior cingulate \\
\hline 50 & Rostral anterior cingulate posterior \\
\hline 51 & Caudal anterior cingulate \\
\hline 52 & Subgenual anterior cingulate \\
\hline 53 & Rostral anterior cingulate mid posterior \\
\hline 54 & Mid anterior cingulate \\
\hline 55 & Supplementary motor area \\
\hline 56 & Cingulate gyrus, posterior division \\
\hline 57 & Precuneus cortex \\
\hline 58 & Cuneal cortex \\
\hline 59 & Supracalcarine cortex \\
\hline 60 & Intracalcarine cortex \\
\hline 61 & Lingual gyrus \\
\hline 62 & Occipital pole \\
\hline 63 & Occipital pole \\
\hline 64 & Lingual gyrus \\
\hline
\end{tabular}

Table 2. Continued

\begin{tabular}{|c|c|c|c|c|c|c|c|c|c|}
\hline Abbreviation & $x$ & Y & Z & & Name & Abbreviation & $x$ & Y & Z \\
\hline FP_L & -30 & 54 & 20 & 65 & Intracalcarine cortex & IC_R & 6 & -68 & 12 \\
\hline OFP_L & -32 & 58 & -6 & 66 & Supracalcarine cortex & SC_R & 2 & -84 & 12 \\
\hline FO_L & -40 & 30 & -14 & 67 & Cuneal cortex & Cun_R & 4 & -82 & 30 \\
\hline SFG_L & -22 & 22 & 54 & 68 & Precuneus cortex & pCun_R & 4 & -64 & 38 \\
\hline MFG_L & -40 & 20 & 44 & 69 & Cingulate gyrus, posterior division & Cingp_R & 4 & 34 & 36 \\
\hline IFGpt_L & -50 & 30 & 16 & 70 & Supplementary motor area & SMA_R & 4 & -2 & 58 \\
\hline IFGpo_L & -54 & -20 & 46 & 71 & Mid anterior cingulate & $\mathrm{ACCm} \_\mathrm{R}$ & 6 & -2 & 42 \\
\hline PreC_L & -44 & -8 & 52 & 72 & Rostral anterior cingulate mid posterior & ACCrm_R & 6 & 18 & 34 \\
\hline PostC_L & -58 & 32 & 40 & 73 & Subgenual anterior cingulate & ACCsg_R & 4 & 16 & -14 \\
\hline SPL_L & -60 & -48 & 32 & 74 & Caudal anterior cingulate & ACCC_R & 4 & 40 & -2 \\
\hline SMGa_L & -54 & -56 & 26 & 75 & Rostral anterior cingulate posterior & ACCrp_R & 2 & 28 & 18 \\
\hline SMGp_L & -40 & 20 & 4 & 76 & Rostral anterior cingulate & ACCr_R & 20 & 28 & 18 \\
\hline Ang_L & -48 & -4 & 8 & 77 & Ventral medial prefrontal cortex & VMPFC_R & 4 & 50 & -20 \\
\hline Fop_L & -48 & -32 & 20 & 78 & Medial prefrontal cortex & MPFC_ $\bar{R}$ & 6 & 60 & 8 \\
\hline Cop_L & -48 & -4 & 8 & 79 & Dorsal medial prefrontal cortex, posterior division & dMPFCp_R & 4 & 26 & 48 \\
\hline Pop_L & -38 & 4 & 0 & 80 & Dorsal medial prefrontal cortex, anterior division & dMPFCa_R & 4 & 54 & 32 \\
\hline Ins_L & -4 & -2 & 58 & 81 & Lateral occipital cortex, inferior division & LOcci_R & 48 & -78 & -2 \\
\hline Caud_L & -12 & 14 & 8 & 82 & Lateral occipital cortex, superior division & LOccs_R & 40 & -78 & 34 \\
\hline Thal_L & 10 & -18 & 8 & 83 & Occipital fusiform gyrus & OccFG_R & 28 & -76 & -14 \\
\hline Put_L & -20 & -4 & 0 & 84 & Temporal occipital fusiform cortex & TOF_R & 34 & -54 & -16 \\
\hline GP_L & -16 & -2 & -2 & 85 & Temporal fusiform cortex, posterior division & TFCp_R & 36 & -16 & -32 \\
\hline Hipp_L & -28 & -22 & -16 & 86 & Temporal fusiform cortex, anterior division & TFCa_R & 32 & -6 & -42 \\
\hline Amyg_L & -24 & -4 & -18 & 87 & Parahippocampal gyrus, posterior division & pHippp_R & 34 & -32 & -18 \\
\hline NAC_L & -10 & 10 & -8 & 88 & Parahippocampal gyrus, anterior division bi & pHippa_R & 34 & -6 & -34 \\
\hline PIP_L & -48 & -4 & -6 & 89 & Inferior temporal gyrus, temporo-occipital & ITGtp_R & 56 & -54 & -18 \\
\hline $\mathrm{He} \_\mathrm{L}$ & -48 & -18 & 6 & 90 & Inferior temporal gyrus, posterior division & ITGp_R & 56 & -32 & -24 \\
\hline PIT_L & -60 & -22 & 8 & 91 & Inferior temporal gyrus, anterior division & ITGa_R & 50 & -6 & -40 \\
\hline STGa_L & -58 & -4 & -6 & 92 & Temporal pole & $T P \_\bar{R}$ & 40 & 16 & -30 \\
\hline STGp_L & -66 & -26 & 6 & 93 & Middle temporal gyrus, temporo-occipital & MTGto_R & 60 & -52 & 0 \\
\hline MTGa_L & -58 & -2 & -22 & 94 & Middle temporal gyrus, posterior division & MTGp_R & 66 & -22 & 12 \\
\hline MTGp_L & -66 & -22 & 12 & 95 & Middle temporal gyrus, anterior division & MTGa_R & 58 & -2 & -22 \\
\hline MTGto_L & -60 & -52 & 0 & 96 & Superior temporal gyrus, posterior division & STGP_R & 66 & -26 & 6 \\
\hline TP_L & -40 & 16 & -30 & 97 & Superior temporal gyrus, anterior division & STGa_R & 58 & -4 & -6 \\
\hline ITGa_L & -50 & -6 & -40 & 98 & Planum temporale & PIT_R & 60 & -22 & 8 \\
\hline ITGp_L & -56 & -32 & -24 & 99 & Heschl's gyrus (includes $\mathrm{H} 1$ and H2) & $\mathrm{He} \_\mathrm{R}$ & 48 & -18 & -6 \\
\hline ITGtp_L & -56 & -54 & -18 & 100 & Planum polare $r$ & PIP_R & 48 & -4 & -6 \\
\hline pHippa_L & -34 & -6 & -34 & 101 & Brainstem & BrStem & 0 & -26 & -28 \\
\hline pHippp_L & -34 & -32 & -18 & 102 & Nucleus accumbens & Nac_R & 10 & 10 & -8 \\
\hline TFCa_L & -32 & -6 & -42 & 103 & Amygdala & Amyg_R & 24 & -4 & -18 \\
\hline TFCp_L & -36 & -16 & -32 & 104 & Hippocampus & Hipp_R & 28 & -22 & -16 \\
\hline TOF_L & -34 & -54 & -16 & 105 & Globus pallidus & GP_R & 16 & -2 & -2 \\
\hline OccFG_L & -28 & -76 & -14 & 106 & Putamen & Put_R & 20 & -4 & 0 \\
\hline LOccs_L & -40 & -78 & 34 & 107 & Caudate & Caud_R & 12 & 12 & 10 \\
\hline LOcci_L & -48 & -78 & -2 & 108 & Thalamus & Thal_R & 10 & -18 & 8 \\
\hline dMPFCa_L & -4 & 54 & 32 & 109 & Insular cortex & Ins_ $\bar{R}$ & 38 & 4 & 0 \\
\hline dMPFCp_L & -4 & 26 & 48 & 110 & Parietal operculum cortex & Pop_R & 48 & -32 & 20 \\
\hline MPFC_L & -6 & 60 & 8 & 111 & Central opercular cortex & Cop_R & 48 & -4 & 8 \\
\hline VMPFC_L & -4 & 50 & -20 & 112 & Frontal operculum cortex & Fop_R & 40 & 20 & 4 \\
\hline$A C C r \_L$ & -6 & 18 & 34 & 113 & Angular gyrus & Ang_R & 54 & -56 & 26 \\
\hline ACCrp_L & -6 & -2 & 42 & 114 & Supramarginal gyrus, posterior division & SMGP_R & 60 & -48 & 32 \\
\hline ACCC_L & -20 & 28 & 18 & 115 & Supramarginal gyrus, anterior division & SMGa_R & 58 & 32 & 40 \\
\hline ACCsg_L & -2 & 28 & 18 & 116 & Superior parietal lobule & SPL_R & 32 & -50 & 60 \\
\hline ACCrm_L & -6 & 60 & 8 & 117 & Postcentral gyrus & PostC_R & 54 & -20 & 46 \\
\hline ACCm_L & -4 & 50 & -20 & 118 & Precentral gyrus & PreC_R & 44 & -8 & 52 \\
\hline SMA_L & -4 & -2 & 58 & 119 & Inferior frontal gyrus, pars opercularis & IFGpo_R & 54 & 14 & 16 \\
\hline Cingp_L & -4 & -64 & 38 & 120 & Inferior frontal gyrus, pars triangularis & IFGpt_R & -50 & 30 & 16 \\
\hline pCun_L & -4 & -82 & 30 & 121 & Middle frontal gyrus & MFG_R & 40 & 20 & 44 \\
\hline Cun_L & -6 & -74 & 12 & 122 & Superior frontal gyrus & SFG_R & 20 & 18 & 62 \\
\hline$S C \_L$ & -2 & -84 & 12 & 123 & Frontal orbital cortex & FO_R & 40 & 30 & -14 \\
\hline IC_L & -28 & -76 & -14 & 124 & Orbitofrontal pole & OFP_R & 32 & 58 & -6 \\
\hline Ling_R & -2 & -84 & 12 & 125 & Frontal pole & $F P_{-} \bar{R}$ & 30 & 54 & 20 \\
\hline OCCP_L & -8 & -100 & 6 & \multirow{2}{*}{\multicolumn{6}{|c|}{ Parcellation scheme was a modified version of the Harvard Oxford Atlas (see Materials and Methods). Table shows }} \\
\hline OCCP_R & 8 & -100 & 6 & & & & & & \\
\hline Ling_R & 10 & -68 & -2 & & ed to show left anterior to posterior re & wed by right & or to ante & erior regi & \\
\hline
\end{tabular}




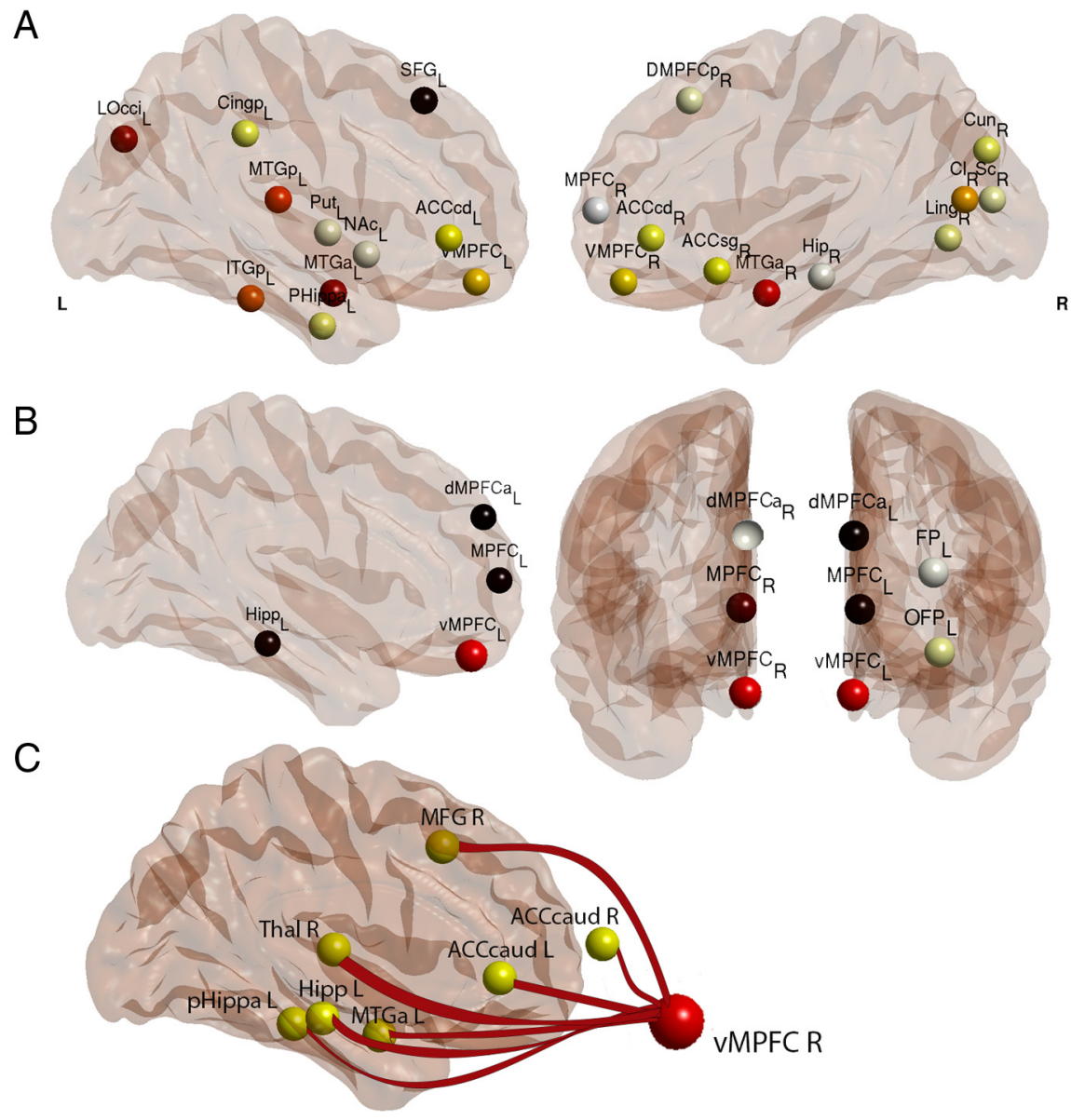

Figure 5. Brain regions with topological properties associated with analgesic response to psychological conditioning. $\boldsymbol{A}$, Brain networks that showed a significant relationship between clustering coefficient and percentage pain decrease in regions represented with randomly colored circles (FDR corrected $T=0.25$ ). Regions involved in cognitive modulation of pain and emotion (SFG, superior frontal gyrus; DMPFC, dorsal medial prefrontal cortex; ACC, anterior cingulate- caudal and subgenual), motivational brain circuitry (MPFC, medial prefrontal cortex; NAc, nucleus accumbens, putamen, globus pallidus), memory (MT, temporal regions; (ingp, posterior cingulate; Hip, hippocampus), and visual circuitry showed higher clustering coefficients in patients that later showed greater psychologically conditioned analgesia. $\boldsymbol{B}$, The centrality (degree) in regions shown in randomly colored circles was positively correlated with greater analgesic response to conditioning (FDR correction $T=0.25$ ). These regions included bilateral MPFC regions (dorsal, ventral, and middle), the hippocampus (Hipp L), the left frontal pole (FPL), and the OFP.C, The right ventral MPFC (vMPFC, shown in red) showed greater functional connectivity in relation with greater analgesic response in regions shown in yellow including bilateral ACC caudal regions (ACCcaud), right middle frontal gyrus (MFG), right thalamus (Thal R), left hippocampus (Hipp L), left parahippocampal gyrus anterior division (paraHippaL), and left middle temporal gyrus anterior (MTGaL). For other abbreviations, see Table 2.

strong local connectivity could be related to stronger conditioning responses.

Although sex did not significantly influence the predictive relationship between clustering and conditioned analgesia, the marginally greater placebo analgesia and positive expectations toward acupuncture treatment in women may be linked with nuanced sex differences in pain perception (Hashmi and Davis, 2014). In addition, women showed markedly greater clustering in resting brain networks as has been observed before in healthy and patient populations (Douw et al., 2011; Tian et al., 2011).

Functional connectivity is associated with functional integration of rhythmic synchronized activity in neuronal ensembles (Sporns et al., 2000a,b). In nonhuman primates, synchronizations of local field potentials between neuronal groups are known to encode task-dependent and content-specific information (Miller and Buschman, 2013). In humans, such neuronal synchronizations of oscillatory signals have been demonstrated at the large scale in functionally specialized but widely distributed cortical regions in relation with normal perceptual brain function (Hipp et al., 2011, 2012). Functional connectivity between distributed but specialized neuronal populations give rise to dynamic interplay between functional integration and segregation, which is in turn suggested to underlie specific perceptual and cognitive states (Sporns et al., 2000a). Network architecture has been less clearly defined in terms of its functional significance and predictive properties. The present finding has implications for network topology as a further level of functional integration in mediating brain processes. Specifically, functional network architecture may serve as a substrate that can facilitate or diminish responses to psychological signals depending on the efficiency of information transfer. Further evidence supporting a role of network topology and behavior has been shown as a relationship between graph metrics and intellectual ability (van den Heuvel et al., 2009) and cognitive performance on a working memory task (Stevens et al., 2012; Langer et al., 2013). This suggests that network topology and community structure may be involved in increasing preparatory resources such as by optimizing information processing within substrate networks that facilitates learning responses to perceptual cues.

This experimental paradigm tests how analgesic response to active or sham treatment is modulated by psychological cues in chronic pain patients. The conditioning paradigm is based on a model of associative learning (Colloca and Benedetti, 2006) that examines how analgesic response to treatment is affected by clinical history of a patient such as good therapeutic alliance between patient and physicians (Kelley et al., 2009; Colloca et al., 2010) or previous treatment outcomes (Miller et al., 2009; Enck et al., 2013). The outcomes hence measure the role of environmental factors and are independent of the therapeutic effects produced by specific pharmacological agents or medical interventions (Miller et al., 2009). While acupuncture may or may not be better than a placebo intervention in treating pain (Miller et al., 2009; Li and Kaptchuk, 2011), we observed psychological factors to have a direct role in modulating pain independent of any direct analgesic effects of the acupuncture intervention. The fact that network architecture predicts psychologically induced analgesia has strong implications for developing predictive tools that can help to isolate analgesic effects specific to active treatments by accounting for psychological factors based on brain network properties. Potentially, we may be able to more objectively characterize effects of active therapeutic agents.

Overall, we demonstrate that chronic pain patients can be expected to show analgesic responses to positive environmental cues associated with clinical treatments. In addition, we provide 


\begin{tabular}{|c|c|c|c|}
\hline Abbreviation & Brain region & $R$ value & $p$ value \\
\hline \multicolumn{4}{|c|}{ Clustering coefficients } \\
\hline SFGL & Superior frontal gyrus left & -0.389 & 0.010847 \\
\hline MTGa R & Middle temporal gyrus anterior right & -0.489 & 0.000999 \\
\hline MTGa L & Middle temporal gyrus anterior left & -0.377 & 0.013695 \\
\hline MTGp L & Middle temporal gyrus posterior left & -0.410 & 0.006986 \\
\hline ITGp L & Inferior temporal gyrus posterior left & -0.390 & 0.010928 \\
\hline LOccil & Lateral occipital cortex superior left & -0.389 & 0.010853 \\
\hline $\mathrm{CLR}$ & Intracalcarine cortex right & -0.414 & 0.006438 \\
\hline VMPFCL & Ventral medial prefrontal cortex left & -0.432 & 0.004235 \\
\hline MPFC R & Medial prefrontal cortex right & -0.370 & 0.015825 \\
\hline DMPFCp_R & Dorsal medial prefrontal cortex post right & -0.353 & 0.002192 \\
\hline Vmpfc R & Ventral medial prefrontal cortex right & -0.479 & 0.001321 \\
\hline$A C C d \mathrm{~L}$ & Caudal anterior cingulate left & -0.405 & 0.007746 \\
\hline$A C C c d R$ & Caudal anterior cingulate right & -0.460 & 0.002138 \\
\hline$A C C s g R$ & Subgenual anterior cingulate right & -0.395 & 0.009532 \\
\hline Cingp L & Cingulate gyrus posterior left & -0.475 & 0.001473 \\
\hline Cun R & Cuneal cortex right & -0.360 & 0.018983 \\
\hline Cun L & Cuneal cortex left & -0.467 & 0.001781 \\
\hline Ling R & Lingual gyrus right & -0.355 & 0.020993 \\
\hline$S C R$ & Supracalcarine cortex right & -0.423 & 0.005179 \\
\hline Put L & Putamen left & -0.397 & 0.009078 \\
\hline Hipp R & Hippocampus right & -0.405 & 0.007723 \\
\hline $\mathrm{NacL}$ & Nucleus accumbens left & -0.357 & 0.025389 \\
\hline \multicolumn{4}{|c|}{ Centrality (degree) } \\
\hline FP_L & Frontal pole left & -0.478 & 0.001362 \\
\hline OFP_L & Orbitofrontal pole left & -0.487 & 0.001061 \\
\hline MPFC_L & Medial prefrontal cortex left & -0.598 & $<0.0001$ \\
\hline VMPFC_L & Ventral medial prefrontal cortex left & -0.478 & 0.001345 \\
\hline MPFC_R & Medial prefrontal cortex right & -0.583 & $<0.0001$ \\
\hline VMPFC_R & Ventral medial prefrontal cortex right & -0.477 & 0.001388 \\
\hline dMPFCa_L & Dorsal medial prefrontal cortex ant right & -0.600 & $<0.0001$ \\
\hline dMPFCa_R & Dorsal medial prefrontal cortex ant right & -0.475 & 0.001466 \\
\hline Hipp_L & Hippocampus left & -0.597 & $<0.0001$ \\
\hline \multicolumn{4}{|c|}{$\begin{array}{c}\text { Functional connectivity } \\
\text { with VMPFC R }\end{array}$} \\
\hline MFG $r$ & Middle frontal gyrus right & -0.34 & 0.0268 \\
\hline MTFGa L & Middle temporal gyrus anterior left & -0.42 & 0.0062 \\
\hline$A C C c d L$ & Caudal anterior cingulate left & -0.4 & 0.0093 \\
\hline$A C C c d L$ & Caudal anterior cingulate left & 0.43 & 0.0044 \\
\hline PHippa L & Parahippocampal anterior gyrus & -0.33 & 0.0305 \\
\hline Hip L & Hippocampus left & -0.32 & 0.0382 \\
\hline Thal R & Thalamus right & -0.36 & 0.0201 \\
\hline
\end{tabular}

Regions that showed a significant relationship between graph metrics and psychologically induced analgesia are listed along with significance values. FDR corrected.

evidence that topological alignments in brain network during the baseline period predict subsequent analgesic response to psychological conditioning. Such local configurations may represent learned changes that increase preparatory resources and facilitate subsequent performance of brain circuits that translate psychological signals into analgesia. Further investigation is required to determine whether functional integration and network architecture are fundamental components of the endogenous analgesic response systems. This finding may potentially serve as a useful predictor of analgesic responses, especially those specific to psychological effects and has implications for new drug discovery and improved clinical care of chronic pain patients.

\section{References}

Baliki MN, Geha PY, Fields HL, Apkarian AV (2010) Predicting value of pain and analgesia: nucleus accumbens response to noxious stimuli changes in the presence of chronic pain. Neuron 66:149-160. CrossRef Medline
Bassett DS, Bullmore ET (2009) Human brain networks in health and disease. Curr Opin Neurol 22:340-347. CrossRef Medline

Beattie KA, Duryea J, Pui M, O’Neill J, Boulos P, Webber CE, Eckstein F, Adachi JD (2008) Minimum joint space width and tibial cartilage morphology in the knees of healthy individuals: a cross-sectional study. BMC Musculoskelet Disord 9:119. CrossRef Medline

Beckmann M, Johansen-Berg H, Rushworth MF (2009) Connectivity-based parcellation of human cingulate cortex and its relation to functional specialization. J Neurosci 29:1175-1190. CrossRef Medline

Benedetti F (2008) Mechanisms of placebo and placebo-related effects across diseases and treatments. Annu Rev Pharmacol Toxicol 48:33-60. CrossRef Medline

Benedetti F, Mayberg HS, Wager TD, Stohler CS, Zubieta JK (2005) Neurobiological mechanisms of the placebo effect. J Neurosci 25:10390-10402. CrossRef Medline

Biswal BB, Mennes M, Zuo XN, Gohel S, Kelly C, Smith SM, Beckmann CF, Adelstein JS, Buckner RL, Colcombe S, Dogonowski AM, Ernst M, Fair D, Hampson M, Hoptman MJ, Hyde JS, Kiviniemi VJ, Kötter R, Li SJ, Lin CP, et al. (2010) Toward discovery science of human brain function. Proc Natl Acad Sci U S A 107:4734-4739. CrossRef Medline

Brown MW, Aggleton JP (2001) Recognition memory: what are the roles of the perirhinal cortex and hippocampus? Nat Rev Neurosci 2:51-61. CrossRef Medline

Bullmore E, Sporns O (2009) Complex brain networks: graph theoretical analysis of structural and functional systems. Nat Rev Neurosci 10:186198. CrossRef Medline

Bullmore E, Barnes A, Bassett DS, Fornito A, Kitzbichler M, Meunier D, Suckling J (2009) Generic aspects of complexity in brain imaging data and other biological systems. Neuroimage 47:1125-1134. CrossRef Medline

Colloca L, Benedetti F (2006) How prior experience shapes placebo analgesia. Pain 124:126-133. CrossRef Medline

Colloca L, Petrovic P, Wager TD, Ingvar M, Benedetti F (2010) How the number of learning trials affects placebo and nocebo responses. Pain 151: 430-439. CrossRef Medline

Colloca L, Klinger R, Flor H, Bingel U (2013) Placebo analgesia: psychological and neurobiological mechanisms. Pain 154:511-514. CrossRef Medline

Craggs JG, Price DD, Verne GN, Perlstein WM, Robinson MM (2007) Functional brain interactions that serve cognitive-affective processing during pain and placebo analgesia. Neuroimage 38:720-729. CrossRef Medline

Craggs JG, Price DD, Perlstein WM, Verne GN, Robinson ME (2008) The dynamic mechanisms of placebo induced analgesia: evidence of sustained and transient regional involvement. Pain 139:660-669. CrossRef Medline

Damasio AR (1996) The somatic marker hypothesis and the possible functions of the prefrontal cortex. Philos Trans R Soc Lond B Biol Sci 351: 1413-1420. CrossRef Medline

Douw L, Schoonheim MM, Landi D, van der Meer ML, Geurts JJ, Reijneveld JC, Klein M, Stam CJ (2011) Cognition is related to resting-state smallworld network topology: an magnetoencephalographic study. Neuroscience 175:169-177. CrossRef Medline

Egúiluz VM, Chialvo DR, Cecchi GA, Baliki M, Apkarian AV (2005) Scalefree brain functional networks. Phys Rev Lett 94:018102. CrossRef Medline

Eippert F, Bingel U, Schoell ED, Yacubian J, Klinger R, Lorenz J, Büchel C (2009) Activation of the opioidergic descending pain control system underlies placebo analgesia. Neuron 63:533-543. CrossRef Medline

Enck P, Bingel U, Schedlowski M, Rief W (2013) The placebo response in medicine: minimize, maximize or personalize? Nat Rev Drug Discov 12: 191-204. CrossRef Medline

Ginestet CE, Nichols TE, Bullmore ET, Simmons A (2011) Brain network analysis: separating cost from topology using cost-integration. PLoS One 6:e21570. CrossRef Medline

Glahn DC, Bearden CE, Caetano S, Fonseca M, Najt P, Hunter K, Pliszka SR, Olvera RL, Soares JC (2005) Declarative memory impairment in pediatric bipolar disorder. Bipolar Disord 7:546-554. CrossRef Medline

Gossec L, Hawker G, Davis AM, Maillefert JF, Lohmander LS, Altman R, Cibere J, Conaghan PG, Hochberg MC, Jordan JM, Katz JN, March L, Mahomed N, Pavelka K, Roos EM, Suarez-Almazor ME, Zanoli G, Dougados M (2007) OMERACT/OARSI initiative to define states of severity 
and indication for joint replacement in hip and knee osteoarthritis. J Rheumatol 34:1432-1435. Medline

Gracely RH, Dubner R (1987) Reliability and validity of verbal descriptor scales of painfulness. Pain 29:175-185. CrossRef Medline

Gracely RH, Kwilosz DM (1988) The Descriptor Differential Scale: applying psychophysical principles to clinical pain assessment. Pain 35:279-288. CrossRef Medline

Hashmi JA, Davis KD (2008) Effect of static and dynamic heat pain stimulus profiles on the temporal dynamics and interdependence of pain qualities, intensity, and affect. J Neurophysiol 100:1706-1715. CrossRef Medline

Hashmi JA, Davis KD (2014) Deconstructing sex differences in pain sensitivity. Pain 155:10-13. CrossRef Medline

Hashmi JA, Baria AT, Baliki MN, Huang L, Schnitzer TJ, Apkarian AV (2012) Brain networks predicting placebo analgesia in a clinical trial for chronic back pain. Pain 153:2393-2402. CrossRef Medline

Hashmi JA, Baliki MN, Huang L, Baria AT, Torbey S, Hermann KM, Schnitzer TJ, Apkarian AV (2013) Shape shifting pain: chronification of back pain shifts brain representation from nociceptive to emotional circuits. Brain 136:2751-2768. CrossRef Medline

Hilz MJ, Devinsky O, Szczepanska H, Borod JC, Marthol H, Tutaj M (2006) Right ventromedial prefrontal lesions result in paradoxical cardiovascular activation with emotional stimuli. Brain 129:3343-3355. CrossRef Medline

Hipp JF, Engel AK, Siegel M (2011) Oscillatory synchronization in largescale cortical networks predicts perception. Neuron 69:387-396. CrossRef Medline

Hipp JF, Hawellek DJ, Corbetta M, Siegel M, Engel AK (2012) Large-scale cortical correlation structure of spontaneous oscillatory activity. Nat Neurosci 15:884-890. CrossRef Medline

Holtzheimer PE 3rd, Mayberg HS (2010) Deep brain stimulation for treatment-resistant depression. Am J Psychiatry 167:1437-1444. CrossRef Medline

Kelley JM, Lembo AJ, Ablon JS, Villanueva JJ, Conboy LA, Levy R, Marci CD, Kerr CE, Kirsch I, Jacobson EE, Riess H, Kaptchuk TJ (2009) Patient and practitioner influences on the placebo effect in irritable bowel syndrome. Psychosom Med 71:789-797. CrossRef Medline

Kellgren JH, Lawrence JS (1957) Radiological assessment of rheumatoid arthritis. Ann Rheum Dis 16:485-493. CrossRef Medline

Kong J, Fufa DT, Gerber AJ, Rosman IS, Vangel MG, Gracely RH, Gollub RL (2005) Psychophysical outcomes from a randomized pilot study of manual, electro, and sham acupuncture treatment on experimentally induced thermal pain. J Pain 6:55-64. CrossRef Medline

Kong J, Gollub RL, Rosman IS, Webb JM, Vangel MG, Kirsch I, Kaptchuk TJ (2006a) Brain activity associated with expectancy-enhanced placebo analgesia as measured by functional magnetic resonance imaging. J Neurosci 26:381-388. CrossRef Medline

Kong J, White NS, Kwong KK, Vangel MG, Rosman IS, Gracely RH, Gollub RL (2006b) Using fMRI to dissociate sensory encoding from cognitive evaluation of heat pain intensity. Hum Brain Mapp 27:715-721. CrossRef Medline

Kong J, Gollub RL, Polich G, Kirsch I, Laviolette P, Vangel M, Rosen B, Kaptchuk TJ (2008) A functional magnetic resonance imaging study on the neural mechanisms of hyperalgesic nocebo effect. J Neurosci 28: 13354-13362. CrossRef Medline

Kong J, Kaptchuk TJ, Polich G, Kirsch I, Vangel M, Zyloney C, Rosen B, Gollub R (2009a) Expectancy and treatment interactions: a dissociation between acupuncture analgesia and expectancy evoked placebo analgesia. Neuroimage 45:940-949. CrossRef Medline

Kong J, Kaptchuk TJ, Polich G, Kirsch I, Vangel M, Zyloney C, Rosen B, Gollub RL (2009b) An fMRI study on the interaction and dissociation between expectation of pain relief and acupuncture treatment. Neuroimage 47:1066-1076. CrossRef Medline

Kong J, Jensen K, Loiotile R, Cheetham A, Wey HY, Tan Y, Rosen B, Smoller JW, Kaptchuk TJ, Gollub RL (2013) Functional connectivity of the frontoparietal network predicts cognitive modulation of pain. Pain 154:459467. CrossRef Medline

Langer N, von Bastian CC, Wirz H, Oberauer K, Jäncke L (2013) The effects of working memory training on functional brain network efficiency. Cortex 49:2424-2438. CrossRef Medline

Latora V, Marchiori M (2001) Efficient behavior of small-world networks. Phys Rev Lett 87:198701. CrossRef Medline
Li A, Kaptchuk TJ (2011) The case of acupuncture for chronic low back pain: when efficacy and comparative effectiveness conflict. Spine 36:181182. CrossRef Medline

Lim SL, O’Doherty JP, Rangel A (2013) Stimulus value signals in ventromedial PFC reflect the integration of attribute value signals computed in fusiform gyrus and posterior superior temporal gyrus. J Neurosci 33: 8729-8741. CrossRef Medline

Meissner K, Bingel U, Colloca L, Wager TD, Watson A, Flaten MA (2011) The placebo effect: advances from different methodological approaches. J Neurosci 31:16117-16124. CrossRef Medline

Miller EK, Buschman TJ (2013) Cortical circuits for the control of attention. Curr Opin Neurobiol 23:216-222. CrossRef Medline

Miller FG, Colloca L, Kaptchuk TJ (2009) The placebo effect: illness and interpersonal healing. Perspect Biol Med 52:518-539. CrossRef Medline

Peat G, McCarney R, Croft P (2001) Knee pain and osteoarthritis in older adults: a review of community burden and current use of primary health care. Ann Rheum Dis 60:91-97. CrossRef Medline

Petrovic P, Dietrich T, Fransson P, Andersson J, Carlsson K, Ingvar M (2005) Placebo in emotional processing-induced expectations of anxiety relief activate a generalized modulatory network. Neuron 46:957-969. CrossRef Medline

Petrovic P, Kalso E, Petersson KM, Andersson J, Fransson P, Ingvar M (2010) A prefrontal nonopioid mechanism in placebo analgesia. Pain 150:59-65. CrossRef Medline

Price DD, Craggs J, Verne GN, Perlstein WM, Robinson ME (2007) Placebo analgesia is accompanied by large reductions in pain-related brain activity in irritable bowel syndrome patients. Pain 127:63-72. CrossRef Medline

Price DD, Finniss DG, Benedetti F (2008) A comprehensive review of the placebo effect: recent advances and current thought. Annu Rev Psychol 59:565-590. CrossRef Medline

Roy M, Shohamy D, Wager TD (2012) Ventromedial prefrontal-subcortical systems and the generation of affective meaning. Trends Cogn Sci 16:147156. CrossRef Medline

Rubinov M, Sporns O (2010) Complex network measures of brain connectivity: uses and interpretations. Neuroimage 52:1059-1069. CrossRef Medline

Scott DJ, Stohler CS, Egnatuk CM, Wang H, Koeppe RA, Zubieta JK (2007) Individual differences in reward responding explain placebo-induced expectations and effects. Neuron 55:325-336. CrossRef Medline

Scott DJ, Stohler CS, Egnatuk CM, Wang H, Koeppe RA, Zubieta JK (2008) Placebo and nocebo effects are defined by opposite opioid and dopaminergic responses. Arch Gen Psychiatry 65:220-231. CrossRef Medline

Sporns O, Tononi G, Edelman GM (2000a) Connectivity and complexity: the relationship between neuroanatomy and brain dynamics. Neural Netw 13:909-922. CrossRef Medline

Sporns O, Tononi G, Edelman GM (2000b) Theoretical neuroanatomy: relating anatomical and functional connectivity in graphs and cortical connection matrices. Cereb Cortex 10:127-141. CrossRef Medline

Stam CJ, Reijneveld JC (2007) Graph theoretical analysis of complex networks in the brain. Nonlinear Biomed Phys 1:3. CrossRef Medline

Stevens AA, Tappon SC, Garg A, Fair DA (2012) Functional brain network modularity captures inter- and intra-individual variation in working memory capacity. PLoS One 7:e30468. CrossRef Medline

Streitberger K, Kleinhenz J (1998) Introducing a placebo needle into acupuncture research. Lancet 352:364-365. CrossRef Medline

Tian L, Wang J, Yan C, He Y (2011) Hemisphere- and gender-related differences in small-world brain networks: a resting-state functional MRI study. Neuroimage 54:191-202. CrossRef Medline

van den Heuvel MP, Stam CJ, Kahn RS, Hulshoff Pol HE (2009) Efficiency of functional brain networks and intellectual performance. J Neurosci 29:7619-7624. CrossRef Medline

van der Kouwe AJ, Benner T, Salat DH, Fischl B (2008) Brain morphometry with multiecho MPRAGE. Neuroimage 40:559-569. CrossRef Medline

van Wijk BC, Stam CJ, Daffertshofer A (2010) Comparing brain networks of different size and connectivity density using graph theory. PLoS One 5:e13701. CrossRef Medline

Wager TD, Rilling JK, Smith EE, Sokolik A, Casey KL, Davidson RJ, Kosslyn SM, Rose RM, Cohen JD (2004) Placebo-induced changes in FMRI in the anticipation and experience of pain. Science 303:1162-1167. CrossRef Medline

Wager TD, Atlas LY, Leotti LA, Rilling JK (2011) Predicting individual 
differences in placebo analgesia: contributions of brain activity during anticipation and pain experience. J Neurosci 31:439-452. CrossRef Medline

Watson A, El-Deredy W, Iannetti GD, Lloyd D, Tracey I, Vogt BA, Nadeau V, Jones AK (2009) Placebo conditioning and placebo analgesia modulate a common brain network during pain anticipation and perception. Pain 145:24-30. CrossRef Medline

Watts DJ, Strogatz SH (1998) Collective dynamics of 'small-world' networks. Nature 393:440-442. CrossRef Medline

Winecoff A, Clithero JA, Carter RM, Bergman SR, Wang L, Huettel SA (2013) Ventromedial prefrontal cortex encodes emotional value. J Neurosci 33:11032-11039. CrossRef Medline

Xia M, Wang J, He Y (2013) BrainNet Viewer: a network visualization tool for human brain connectomics. PLoS One 8:e68910. CrossRef Medline

Zhu D, Gao Y, Chang J, Kong J (2013) Placebo acupuncture devices: considerations for acupuncture research. Evid Based Complement Alternat Med 2013:628907. CrossRef Medline

Zubieta JK, Bueller JA, Jackson LR, Scott DJ, Xu Y, Koeppe RA, Nichols TE, Stohler CS (2005) Placebo effects mediated by endogenous opioid activity on mu-opioid receptors. J Neurosci 25:7754-7762. CrossRef Medline

Zubieta JK, Yau WY, Scott DJ, Stohler CS (2006) Belief or Need? Accounting for individual variations in the neurochemistry of the placebo effect. Brain Behav Immun 20:15-26. CrossRef Medline 